\title{
Assessment of Rational Drug Prescribing Pattern in Geriatric Patients in Hyderabad Metropolitan
}

\author{
Anitha Nandagopal*, Anupama Koneru, Arifa Rahman, Md Khalid Pasha, Md Khaja Yaqub Ali
}

Department of Pharmacology, Sultan-ul-Uloom College of Pharmacy, Banjara Hills, Hyderabad, Telangana, INDIA.

\begin{abstract}
Background: Polypharmacy and inappropriate prescriptions are prominent prescribing issues among geriatric patients. Beer's criteria, Drug-Drug interaction of common OTC drugs and WHO essential drug lists have been developed to assist in the reduction of potentially inappropriate medications prescribed to geriatric patients. Objective: This study was carried out to find out the rational prescription of drugs in the geriatric patients and to also investigate Polypharmacy. Methodology: A retrospective study on rational drug prescribing pattern in geriatric patient was carried out using prescriptions issued to the geriatric patients, 65 years and above, attending the outpatient and inpatient department of various hospitals and clinics of Hyderabad. A total of 125 prescriptions were collected and analyzed by using Beer's Criteria. Results: In this study we observed that almost all prescriptions were with polypharmacy. Only $3 \%$ of drugs were prescribed on generic names and remaining all is on brand names. $11(13.75 \%)$ prescriptions had one or more potentially inappropriate medicines from Beer's Criteria and in $9(11.25 \%)$ prescriptions drug-drug interactions were ascertained according to drugdrug interactions of common OTC drugs. Conclusion: Prevalence of polypharmacy was high which is usually unavoidable in the geriatric patients. Extending clinical pharmacist services can significantly improve rational use of medicines in geriatric population.
\end{abstract}

Key words: Geriatric population, Rational drug use, Essential drug list, Polypharmacy, Beer's criteria.

\section{INTRODUCTION}

One of the most pressing problems facing public health providers and administrators in many countries is the rational use of the drug. ${ }^{1}$ Therefore, the concept of rational drug use during the past few years has been the theme of various national and international gatherings. Various studies conducted in developing as well as in developed countries during past few years regarding the safe and effective use of drugs show that irrational drug use is a global phenomenon and only few prescriptions justify rational use of drugs. ${ }^{2}$

World Health Organisation (WHO) convened a conference on "Rational Drug Use" in Nairobi in 1985 defined rational drug use as follows: The rational use of drug requires that patients receive medications appropriate to their clinical needs, in doses that meet their own individual requirements for an adequate period and at the lowest cost to them and their community. The five important criteria for rational drug use are accurate diagnosis, proper prescribing, correct dispensing, suitable packing and patient's adherence. The prescriber should make an accurate diagnosis and prescribe rationally, and the pharmacist should ensure that effective form of the drug reaches the right patient in prescribed dosage and quantity, with clear instructions on its appropriate use. Competent and qualified pharmacist should be trained for dispensing and for giving proper instruction to the patients on safe and effective usage of drugs. ${ }^{3}$

Medicines are used rationally when patients
DOI: 10.5530/ijopp.10.3.37

Address for correspondence: Anitha Nandagopal, Professor and HOD, Department of Pharmacology, Sultan-ul-Uloom College of Pharmacy, Banjara Hills, Hyderabad-500 034, Telangana, INDIA.

Phone no: 09959971590 E-mail: anirajan_76@yahoo.co.in

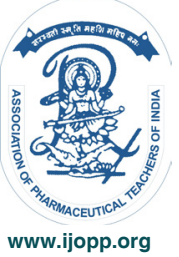


receive the appropriate medicines, in doses that meet their own individual requirements, for an adequate period and at the lowest cost both to them and their community. ${ }^{4}$ Rational medication prescribing dictates that the fewest medications be used achieve the therapeutic goals as determined by clinician and patients. Multiple medications not only add to the cost and complexity of therapeutic regimens, but also play patients at greater risk for adverse drug reactions and drug-drug interactions. ${ }^{5}$

Rational drug use assumes significance in elderly as they use more prescribed and over the counter drugs than the younger populations. A disproportionate number of elderly people suffer from chronic and degenerative pathology, leading in turn to a demand for more medication. However, knowledge about the efficacy and safety of many drugs is often sparse for the frail for the elderly because they are generally excluded from clinical trials. While being the major consumers and the greatest beneficiaries of modern drug therapy, elderly patients are particularly vulnerable and most at risk of suffering adverse drug reactions. ADR's in the elderly have been characterized as "a major modern epidemic". ${ }^{6}$

There is 3-7 times greater incidence of ADR's in the elderly as compared to the age group 20-29 years. Up to $30 \%$ of drug in take may result in ADR's contributing to $10 \%$ of hospital admissions in elderly. The decline is physiological in the geriatric population results in poor compensation and recovery from ADR's. Elderly population should receive drugs only for well defined indications at the lowest effective doses. ${ }^{7}$

The objective of study was to assess the Rationality of drug prescribing for geriatric patients, according to Beer's criteria, Drug-Drug interaction of common OTC drugs and WHO essential drug list.

\section{OBJECTIVES OF THE STUDY}

1. To find out the rational prescription of drugs in the geriatric patients.

2. To investigate the occurrence of Polypharmacy.

\section{MATERIALS AND METHODS}

\section{Study design}

A retrospective study on rational drug prescribing pattern in geriatric patient was carried out using prescriptions prescribed to the geriatric patients, 65 years and above attending the outpatient and inpatient department of various hospitals and clinics of Hyderabad. The prescriptions were collected from for a period of 3 months.

\section{Sample size and collection}

A total of 125 prescriptions of patient $\geq 65$ years old emanating outpatient and inpatient departments of hospitals and clinics were consequently selected. This sample size exceeds the minimum of 100 prescriptions suggested by WHO was employed to enhance the reliability of the results.

\section{Data collection}

A data collection format was designed to aid collation of data. Data on age, sex, and number of medicines per prescription, percentage encounter of these drugs as generics or brand names, percentage occurrence of injections, percentage occurrence of antibiotics, percentage of essential drugs, dose and duration of the prescribed drugs were obtained.

Occurrence of irrational prescribing for these geriatric patients and various drug-drug interactions were ascertained using parameters such as Beer's Criteria, WHO essential drug list and Drug-Drug interactions of common OTC drugs.

\section{Procedure for calculating prescribing indicators ${ }^{8}$}

Average number of drugs per encounter was calculated by dividing the total number of drugs prescribed by the number of prescriptions surveyed. Percentage of drugs prescribed by generic name was determined by dividing the number of drugs prescribed by generic name by the total number of drugs, which was then multiplied by 100. Percentage of encounters with an antibiotic and injection prescription was calculated by dividing the number of patient encounters during which an antibiotic or an injection was prescribed by the total number of encounters surveyed, which was then multiplied by 100 respectively. Percentage of drugs prescribed from the WHO essential drug list was determined by dividing the number of products prescribed from the essential drug list by the total number of drugs prescribed, and then multiplied by 100 .

\section{RESULTS}

\section{Demographic Data}

The demographic characteristics of the geriatric patients were shown in the Table 1 . Out of 125 prescriptions studied, $59(47.2 \%)$ belong to males and the rest 


\begin{tabular}{ccc}
$\begin{array}{c}\text { Table 1: Demographic characteristics of the geriatric patients }(\mathbf{N}=125) \\
\text { Characteristics }\end{array}$ & Percentage\% \\
\hline AGE(YEARS) & 57 & \\
$60-65$ & 33 & 45.6 \\
$66-70$ & 12 & 26.4 \\
$71-75$ & 17 & 9.6 \\
$76-80$ & 6 & 13.6 \\
$81-85$ & & 4.8 \\
SEX & 59 & \\
MALES & 66 & 47.2 \\
FEMALES & & 52.8 \\
\hline
\end{tabular}

$66(52.8 \%)$ belong to females, giving a male to female ratio of 0.94:1.05. Maximum numbers of prescriptions were seen in the age group of 65-70 years followed by $71-75$ years. 17 prescriptions were prescribed at the age group of $81-85$ and 6 prescriptions were at the age group of 86-90. These details were shown in Table 1.

\section{Prescription of medicines according to therapeutic groups}

The distributions of the medicines prescribed into their therapeutic groups were shown in the Figure 1. AntiDiabetics were the most prescribed medicines $(21.90 \%)$ with metformin being the most prescribed anti diabetic agent. Antibiotics ranked second (17.78\%) with ampicillin being the most prescribed antibiotic. Cardiovascular Drugs such as anti-hypertensive, antihyperlipidaemics and calcium channel blockers ranked third (16.26\%) with digoxin being most prescribed cardiovascular drug. Gastrointestinal Drugs ranked fourth (13.017\%). Vitamins and Minerals were prescribed in $12.58 \%$ and diuretics were prescribed in $9.11 \%$ prescriptions. Psychotherapeutic drugs and injectables were prescribed in $4.77 \%$ and $2.38 \%$ prescriptions respectively.

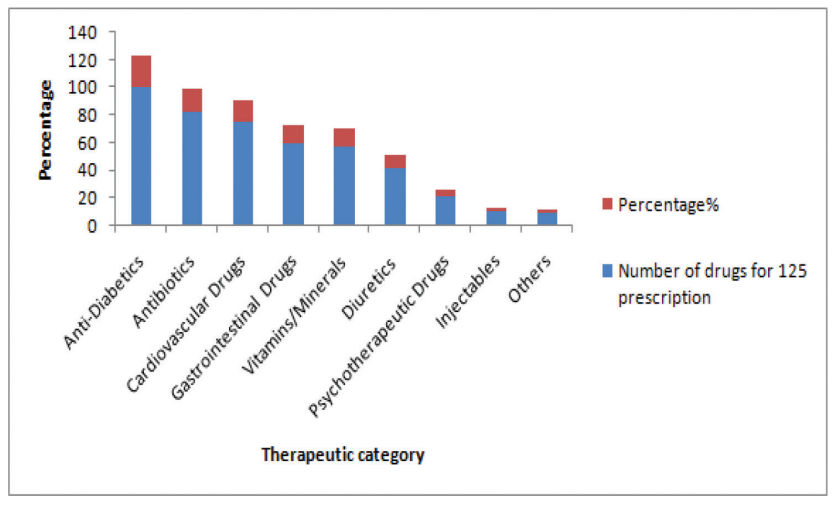

Figure 1: Prescription of medicines according to therapeutic groups $(\mathrm{N}=125)$.

\section{Who Prescribing Indicators}

The prescribing indicator values obtained were shown in Table 2.

\section{Average number of medicines per prescription}

The average number of medicines per prescription was 7.02; the range being 1 to 4 medicines.

\section{Percentage of medicines prescribed by generic names}

A total of $15(3.25 \%)$ of the drugs were prescribed in their generic names.

\section{Percentage of encounters with an antibiotic prescribed}

$82(17.78 \%)$ prescriptions had one or more antibiotics prescribed.

\section{Percentage of encounters with an injection prescribed}

A total of $11(2.38 \%)$ of the prescriptions had at least one injection prescribed along with other drugs.

\section{Percentage of drugs prescribed from the WHO Essential Drug List}

About $33 \%$ of the medicines were prescribed according to WHO essential drug list.

\section{Extent of potentially inappropriate medicines}

Of the 125 prescriptions studied, $11(13.75 \%)$ had one or more potentially inappropriate medicines from Beer's Criteria. In $9(11.25 \%)$ prescriptions drug-drug interactions were ascertained according to drug-drug interactions of common OTC drugs.

Indian Journal of Pharmacy Practice, Vol 10, Issue 3, Jul-Sep, 2017 


\begin{tabular}{|c|c|c|}
\hline Prescribing Indicator & Values Obtained & WHO Standard \\
\hline Average number of drugs per prescription. & 7.02 & $1.6-4.8$ \\
\hline$\%$ of drugs prescribed as generics & 3.25 & $100 \%$ \\
\hline$\%$ of antibiotic per prescription & 17.78 & $20-26.8 \%$ \\
\hline$\%$ of injections per prescription & 2.38 & $13.4-24.1 \%$ \\
\hline
\end{tabular}

\section{DISCUSSION}

Prescribing patterns of drugs reflects the clinical judgment of the clinicians. Our study revealed poly pharmacy in geriatric patients with an average number of drugs per prescription being 7.02. This deviates from the WHO standards of 1.6 to 4.8. Poly pharmacy unfortunately is very common in India and some other countries. ${ }^{9}$ It results in increased cost of treatment, which may lead to non-adherence by patients as they have more medicines than they can cope with. It also increases the risk of significant adverse drug interaction.

It may be advisable for pharmacists to discuss the availability at once daily, single tablet, fixed dose combinations to reduce pill burden and improve adherence. $^{10}$

This study revealed the use of many therapeutic groups among the elderly. Anti-diabetics were the most frequently prescribed, with metformin ranking as the highest of them. The next most frequently prescribed group is antibiotics. Ampicillin was found to be the highly prescribed drug among antibiotics.

The third most prescribed therapeutic group is the cardiovascular drugs among which anti-hypertensive most frequently were prescribed. The incidence of hypertension in the geriatric population is very high and is significant determinant of cardiovascular risk in this group. The tendency for blood pressure to increase with age may depend on environmental factors such as diet, stress and inactivity. Senescent changes in the cardiovascular system leading to decreased vascular compliance and decreased baroreceptor sensitivity contribute to rising blood pressure. The hallmark of hypertension in the elderly is increased vascular resistance. ${ }^{11}$

Gastro intestinal drugs were the fourth most prescribed medicines. Vitamins/Minerals were the fifth most prescribed medicines. The high occurrence of vitamin and other health supplements in the prescription were not surprising as many people don't consume an optimal amount of all vitamins by diet alone. Pending strong evidence of effectiveness from randomized trials, it appears prudent for adults to take vitamin supplements. Physicians should make specific efforts to learn about their patient's use of vitamins to ensure that they take only the vitamins that they should. ${ }^{12}$

$3.25 \%$ of medicines prescribed were generics. This finding is like those of previous studies, ${ }^{13}$ but falls short of WHO recommendation of $100 \%$. This implies that the prescribers are not complying with the recommendations of WHO prescriber indicator. Prescribing by generic name allows flexibility of stocking and dispensing various brands of a drug that are cheaper than and as effective as proprietary brands. This is the basis of essential drug list use. ${ }^{14}$ Moreover, from our study we found that only $33 \%$ of prescribed drugs were fall on WHO essential drug list which need more attention of the clinicians.

It is worth noting that prescribing of antibiotics fell within the WHO recommended range of 20-26, while prescribing of injections was lower than the recommended range of $\mathrm{WHO}(13.4-24.0 \%)$. A lower rate of injection prescribing $(7.5 \%)$ has also been reported by Akande and Ologe. ${ }^{15}$

In many prescriptions drugs were prescribed by brand names where less expensive generic equivalents are available. This could be because the prescribers are more conversant with the brand names than generic names of the drug products. Also pressure from the medical representative of the branded products to prescribe their own brand may have contributed immensely to this high rate.

Of great importance is the occurrence of inappropriate prescribing among the elderly observed in this study. This form of irrational drug use brings about high economic burden on the patients who must buy the branded products at a higher cost than a generic with the same bioequivalence.

About $13.75 \%$ of prescriptions met Beer's criteria for inappropriate prescribing in the elderly and $11.25 \%$ of prescriptions met drug-drug interactions of common OTC drugs. The reasons for inappropriate prescribing 
may be partly due to relatively weak evidence based guidelines for appropriate prescribing in elderly patients and existence of justifying exceptions to the rules in individual patients. ${ }^{16}$

Inappropriate medication uses in patients, 65 years and above has been linked to many adverse drug reactions; poor physical functioning and excess health care use. ${ }^{17}$ Interventions could target more appropriate drug selection by physician to elderly patients. In most of the prescriptions dose and duration were not mentioned which also justifies inappropriate prescribing.

\section{CONCLUSION}

Prescribing for the elderly was found to be suboptimal and there was occurrence of inappropriate prescribing. This calls for caution on the part of prescribers and pharmacist alike and the need for awareness of tools that can be used by practitioners for detecting drug therapy problems. More studies are required on the pattern of inappropriate prescribing over a long period of time and on intervention programs to reduce potentially adverse health outcomes in elderly patients most at risk in the area where this study was undertaken.

\section{ACKNOWLEDGEMENT}

We thank Sultan-ul-Uloom Education Society for providing us the facilities to carry out the research work.

\section{CONFLICT OF INTEREST}

No conflict of interest is declared.

\section{ABBREVIATION USED}

OTC: Over the Counter; WHO: World Health Organization; ADR: Adverse Drug Reaction.

\section{REFERENCES}

1. Thomas M, Alexander B, Tony S, Andrei Z. Guidelines for implementing drug utilization review programs in hospital. Arlington, VA/Moscow, Russia. January 1997.

2. Dr. Sneha Ambwani and Dr. Mathur, Health Administrator volume XIX Number 1:5-7.

3. Alam K, Mishra P, Prabhu M, Shankar PR, Plalain S, Bhandari RB et al. A study on rational drug prescribing and dispensing in outpatients in a tertiary care teaching hospital of western Nepal. Kathmandu University Medical Journal. 2006;4(4):16, 436-43.

4. The Rational use of drugs, report on the conference of experts Nairobi sponsored by WHO Geneva. 1985.

5. Good CB. Polypharmacy in elderly patients with diabetes. Diabetes Spectrum. 2002;15(4):240-8.

6. Strand J, Rokstad K. Elderly patients in general practice: diagnosis, drugs and inappropriate prescriptions. A report from the More and Romsdal prescription study. Family Practice. 1999;16(4):380-8.

7. Ramesh warnath C, Alok KS, Gambhir IS. Rational Drug Therapy in Elderly. Journal of the Indian Academy of Geriatrics. 2005;2:82-8.

8. Debalto D, Gobe Z, Teklemariam S: A base line survey of prescribing indicators and factors influencing prescribing in southern Ethiopia. Ethiopian Journal of Health Development. 1991;11(3):263-7.

9. Fletcher RH, Fairfield KM. Vitamins for Chronic Disease Prevention in Adults Clinical Applications. JAMA. 2002;287(23):3127-9.

10. Yousuf KB, Balogun OB. Pattern of drug utilization among hypersensitive in a Nigerian teaching hospital. Pharmocoepidemiology and drug Safety. 2004;14(1):69-74.

11. Adibe MO, Aguwa CN, Ukwe CV, Okonte JM, Udeogaranya PO. Outpatient utilization of antidiabetic drugs in the south-eastern Nigeria. International Journal of Drug Development and Research. 2009;1(1):27-36.

12. Akande TM, Ologe MO. Prescription pattern at a secondary Health Care Facility in Illorin, Nigeria. Annals of African Medicine. 2007;9(4):186-9.

13. Curtis LH, Ostbye T, Sendersky V, Hutchison S, Dans PE, Wright A, et al. Inappropriate prescribing for elderly Americans in a large outpatient population. Archives of Internal Medicine. 2004;164(15):1621-5.

14. Goulding MR. Inappropriate medication prescribing for elderly ambulatory care patients. Archives of Internal Medicine. 2004;164(3):305-12.

15. Akande TM, Ologe MO. Prescription pattern at a secondary Health Care Facility in Illorin, Nigeria. Annals of African Medicine. 2007;9(4):186-9.

16. Uchenna $\mathrm{IH}$, Adebiyi $\mathrm{OO}$. Prescribing patterns and inappropriate use of medications in elderly outpatients in a tertiary hospital in Nigeria. Tropical Journal of Pharmaceutical Research February. 2011;10(1):19-25.

17. Febin A, Gladis V, Joseph CM, Phebina MJ, Gloria KS. Drug utilization pattern among geriatric patients in a tertiary care teaching hospital. Asian Journal of Pharmaceutical and Clinical Research. 2015;8(6):1991-4. 